Mar a Jun 2021 - v.11 - n.2

\title{
A gestão ambiental nas instituições de ensino superior
}

A crise política e econômica atual faz com que o interesse em proporcionar uma visão sobre a importância da sustentabilidade através da Gestão Ambiental nas Instituições de Ensino Superior seja imprescindível. O objetivo deste trabalho foi identificar ações ligadas à eficiência de insumos, adotadas pelas Instituições de Ensino Superior (IES) em funcionamento, que podem ser replicadas. Para isso, utilizou-se de pesquisa bibliográfica e qualitativa. Os resultados demonstram que as IES dever-se comprometer a informar a comunidade sobre as medidas de proteção de forma transparente e fornecer a educação, treinamento e incentivo aos seus colaboradores em relação à preservação do meio ambiente e das possíveis soluções de problemas ambientais. E de forma participativa antes da aquisição de insumos verificar com a comunidade as reais necessidades dos mesmos, dessa forma os efeitos ambientais ocasionados por materiais e equipamentos devem ser levados em consideração pela instituição antes da compra. Devendo prioritariamente as IES desempenhar atividades ambientais relevantes e garantir a liberdade na pesquisa e ensino, conscientizando a comunidade de sua responsabilidade social e das consequências ambientais que certos resíduos podem ocasionar. Por último, enfatizar-se a importância de se preservar os recursos naturais para as gerações presentes e futuras, e a promoção da busca de auxilio de colaboradores e discentes, com a finalidade de minimizar as consequências negativas na pesquisa e no ensino institucional, garantindo assim, a maior oferta de trabalhos acadêmicos na área ambiental e cursos que tratam das questões ambientais, em cooperação com outros centros de pesquisas.

Palavras-chave: Política ambiental; Meio ambiente; Gestão sustentável; Sustentabilidade; Ecoeficiência.

\section{Environmental management in higher education institutions}

The current political and economic crisis makes the interest in providing a view on the importance of sustainability through Environmental Management in Higher Education Institutions to be essential. The objective of this work was to identify actions related to the efficiency of inputs, adopted by the Higher Education Institutions (IES) in operation, which can be replicated. For this, we used bibliographic and qualitative research. The results demonstrate that HEls must commit themselves to inform the community about protection measures in a transparent manner and provide education, training and incentives to their employees regarding the preservation of the environment and possible solutions to environmental problems. And in a participatory way before purchasing inputs, check with the community their real needs, so the environmental effects caused by materials and equipment must be taken into account by the institution before purchase. As a priority, HEls must carry out relevant environmental activities and guarantee freedom in research and teaching, making the community aware of their social responsibility and the environmental consequences that certain waste can cause. Finally, emphasize the importance of preserving natural resources for present and future generations, and promoting the search for help from employees and students, in order to minimize the negative consequences in research and institutional education, thus guaranteeing, the greatest offer of academic works in the environmental area and courses that deal with environmental issues, in cooperation with other research centers.

Keywords: Environmental policy; Environment; Sustainable management; Sustainability; Eco-efficiency.

Topic: Desenvolvimento, Sustentabilidade e Meio Ambiente

Reviewed anonymously in the process of blind peer.

Francenilde da Silva Feitosa (ib

Universidade Norte do Paraná, Brasil

http://lattes.cnpq.br/6244322090047487

http://orcid.org/0000-0002-3779-6490

francenilde19@gmail.com

Neidiara da Silva Feitosa (iD

Universidade Norte do Paraná, Brasil

http://lattes.cnpq.br/8056138395761376

http://orcid.org/0000-0003-3426-3004

yara.feitosa48@gmail.com

Francisco Rubens Feitosa Júnior (iD

Universidade Federal do Sul da Bahia, Brasil

http://lattes.cnpq.br/1603533849627421

http://orcid.org/0000-0001-7793-2138

rubensjr21@hotmail.com
Received: 20/03/2021

Approved: 10/04/2021

\section{Referencing this:}

FEITOSA, F. S.; FEITOSA, N. S.; FEITOSA JÚNIOR, F. R.. A gestão ambiental nas instituições de ensino superior. Natural Resources, v.11, n.2, p.51-57, 2021. DOI: http://doi.org/10.6008/CBPC22379290.2021.002.0007

DOI: 10.6008/CBPC2237-9290.2021.002.0007 


\section{INTRODUÇÃO}

O Brasil aderiu a Agenda 21, com a finalidade de promover uma política econômica que tenha a preservação dos recursos naturais e o desenvolvimento sustentável como princípio fundamental na tomada de suas decisões (BRUSEKE, 1996). O consumo sustentável no Brasil é fundamentado na legislação nacional que visa o fornecimento a sociedade de recursos naturais, assim como a redução de efeito negativo sobre a saúde humana e sobre o meio ambiente.

Da mesma forma, o artigo 225 da Constituição Federal (1988) ressalta a importância de se preservar o meio ambiente para futuras gerações. A diminuição do consumo de energia elétrica e água são fundamentais na gestão ambiental e em 2015 o Ministério do Planejamento, Orçamento e Gestão publicou a Portaria № 23, que visa conscientizar os órgãos e entidades da administração pública federal em relação ao uso sustentável de seus recursos.

Neste contexto, as ações conjuntas dos diversos setores da administração pública federal são importantes para que juntamente com as Instituições de Ensino Superior (IES) possam formar a opinião social, e apontar alternativas de usos sustentáveis dos recursos não renováveis. Pois, o papel social das IES em relação à gestão ambiental, não pode ser considerado apenas um processo administrativo, limitado pelos interesses econômicos (BRANDLI et al., 2012).

Dessa forma, o desenvolvimento sustentável depende da educação, para a conscientização social de maneira que cada membro possua instrução a respeito da sustentabilidade ambiental (MAYOR, 1998). No Brasil, segundo o Censo da Educação Superior (INEP, 2018) são aproximadamente 8.450 .755 discentes no ensino superior. Desses, 6.394.244 são de cursos presenciais, sendo ofertados 37.962 cursos de graduação, distribuídos em 2.537 instituições (299 públicas e 2.238 particulares), e as Instituições de Ensino Superior oferecem oportunidades para a pratica da educação e da gestão ambiental.

No entanto, um impedimento para uma prática sustentável nas Instituições de Ensino Superior é de ordem financeira, a falta de recursos ocasionam em alguns casos a utilização de insumos que não são recicláveis (NOGUEIRA, 2014), ou geram resíduos em grandes quantidades que acabam sendo descartado em locais inapropriados (EL FARO et al., 2012). É abrangente a escassez de recursos e impedimentos, porém muitas IES conseguiram tomar medidas para melhorar seu nível de sustentabilidade (WOLSKI, 2009).

A ação de discentes, docentes, auxiliares administrativo, e demais funcionários que buscam a sustentabilidade, resulta na conscientização ambiental e contribui para promover uma instituição com melhores uso e ocupação de seus espaços, através da promoção continua da diminuição de resíduos nos ambientes dos IES e restaurantes que se situam ao redor da instituição (OSIBANJO et al.,2007; STEP, 2011; WIDMER et al., 2005). E os grupos de estudo, projetos e pesquisa são as principais alternativas para se discutir e descrever a realidade vivenciada na instituição e isso repercute principalmente na economia de recursos e soluções que abarcar as áreas relacionadas ao setor de ensino superior, e as quais podem também gerar tentativas de superar os impedimentos acima mencionados (SHARP, 2002).

Dessa forma a proposta deste trabalho é abordar a eficiência da gestão ambiental nas Intuições de Ensino Superior, como alternativa para se chegar a um desenvolvimento sustentável. A relevância deste 
estudo justifica-se devido o tema sustentabilidade se amparar em acordos internacionais e em normativas nacionais que precisam ser avaliadas, e as Instituições de Ensino Superior exerce um papel fundamental na sociedade.

E com isso, verifica-se que a proteção ambiental dentro do funcionamento das IES é integrada, os aspectos ambientais são cada vez dinâmicos, e cada membro da comunidade tornando-se responsável pela proteção ambiental em suas próprias áreas (ROGERS et al., 2001), porque a responsabilidade não reside apenas em um grupo de especialistas, pois, o conhecimento especializado nas áreas individuais pode ser utilizado para se chegar à sustentabilidade ambiental.

\section{METODOLOGIA}

A presente pesquisa se fundamentou em uma abordagem qualitativa que segundo Zanella (2012), caracteriza-se por conhecimentos teórico-empíricos que através da subjetividade, gera resultados replicáveis. Os procedimentos utilizados neste trabalho foi inicialmente a revisão da literatura e em seguida a revisão documental. A revisão da literatura foi realizada conforme Lakatos et al. (2003), por meio de uma síntese de fontes secundárias publicadas em jornais, revistas, livros, monografias, artigos, dissertações, teses e outros. Com o ensejo de obter uma concepção fundamentada sobre o estado atual dos conhecimentos a respeito da problemática da pesquisa. Posteriormente realizou-se a leitura dos resumos para identificar ações referentes à gestão ambiental implementadas em Instituições Públicas de Ensino Superior. A pesquisa documental foi realizada segundo com Gil (2002), sendo utilizada para identificar o consumo e os insumos mais utilizados.

\section{DISCUSSÃO TEÓRICA}

\section{Alternativas básicas da promoção da sustentabilidade ambiental nas instituições de ensino superior (IES)}

As diretrizes ambientais norteiam as decisões relacionadas ao meio ambiente nas Instituições de Ensino Superior (IES). A política ambiental determina, desse modo, os objetivos gerais e os princípios básicos que promove a sustentabilidade ambiental, e através das diretrizes representam o compromisso voluntário em relação à proteção do meio ambiente, tanto para a comunidade interna como para a comunidade externa a Instituição (BOFF, 2012; BRANDLI et al., 2012).

$\mathrm{O}$ procedimento de registrar e publicar internamente todos os requisitos legais, administrativos e ambientais é recomendado em todas as instituições que visam chegar a ecoeficiência (RINGSTROM et al., 2011). Embora o cumprimento dos requisitos das diretrizes ambientais seja uma realidade em muitos setores públicos, existem ainda instituições que não estão cumprindo-as (SALGADO, 2006).

Dessa forma uma avaliação ambiental por meio de auditoria pode trazer à baila a visão geral da relevância ambiental que os agentes responsáveis pela Instituição tenham dado durante sua gestão (BRANDLI et al., 2012; JORGE et al., 2015). Por meio dos fluxos de energia, de estoques de insumos de entrada e saída, é possível avaliar a relevância e implementar alternativas para redução do consumo de recursos institucional (GARCIA et al., 2016). 
Com base na ISO 14040, é possível fazer a avaliação ambiental usando algumas etapas como: a definição de objetivo; inventário; avaliação de impacto e interpretação (PRYSHLAKIVSKY et al., 2013). Esse tipo de análise visa fornecer uma visão geral de material e fluxos de energia, e parte incialmente por meio da avaliação nos edifícios da instituição, para que na sequência os resultados sejam ajustados para toda a instituição. O objetivo é identificar processos que afetam a IES, a fabricação ou geração de resíduos de impacto ambiental, que se não identificados, a omissão destes irá comprometer os resultados obtidos na análise ambiental realizada na instituição.

Outro processo de relevante importância é a Emissões de $\mathrm{CO}_{2}$ que tem suas emissões relacionadas à energia, tráfego e água residual nas IES. Muitas intuições contribuem com consumo de energia e derivados de combustíveis fosseis que acelera o Efeito Estufa (UNHABITAT, 2016), pois não se atentam para indicadores de Emissões de $\mathrm{CO}_{2}$ na sua gestão ambiental. Por isso a IFS deve propor em seus objetivos com base nas diretrizes ambientais para obter resultados de relevância para a sociedade (BOCKEN et al., 2014; DUTTA et al., 2016; FREITAS et al., 2012).

Uma boa gestão ambiental serve para implementar os requisitos descritos na legislação e cumprir medidas para cada um dos itens descritos nas leis. Dessa forma a produção de relatório ambiental, envolve especificar ao público e membros institucionais a respeito das medidas implementadas, e as que serão realizados durante a gestão. E deve-se buscar compor um relatório com informações aprofundadas, e bem fundamentadas nos dados obtidos para que assim possa ser avaliado por todos os interessados. Podendo ser disponibilizado por meio eletrônico e mídias digitais (ALONSO-ALMEIDA et al., 2015).

E isso auxiliar as IES a promover a capacitação e treinamentos, além de cursos utilizando aspectos de proteção ambiental empregado pela própria instituição. Para ressaltar a participação da pesquisa, ensino, discentes, e da administração universitária na promoção da sustentabilidade, a instituição, pode possibilita a coleta de dados relativos à própria instituição, que poderá discutir e resolver problemas através de grupos formados para essa finalidade.

Então, torna-se evidente que para mudar a realidade que vivemos com tanta produção de resíduos, toda a sociedade deve participar. Destacam-se, porém que as alternativas pode levar a uma melhoria da situação ambiental ou não, pois cada instituição pode apresentar situações especificas que requer medidas e intervenções que pode perdurar por muitos anos para reverter impactos ambientes existentes. Pois, iniciativas anteriores que não seguiam as diretrizes ambientais tornam os danos intrínsecos e as soluções para problemas ambientais elaborados pela falta de planejamento não implementadas (NOGUEIRA, 2014).

O que repercute em um trabalho de reversão de danos ambientais que exigirá maior demanda de tempo, podendo chegar a anos, principalmente para conscientizar os colaboradores sobre a necessidade de novas formas de atitude em relação as suas atividades (AGUIAR, 2015). Mesmo que membros institucionais apresente conhecimento de aspectos ambientais em seus currículos e atividade, nessa situação é imprescindível a criação da função de coordenador de Gestão Ambiental para melhor orientar a todos e responder pelas futuras intervenções institucionais que promova a sustentabilidade (BRIDGESTOCK, 2012).

O Gestor pode também propor o desenvolvimento por meio de uma gestão participativa, trazendo 
à comunidade acadêmica objetivos, implementação das medidas, e os resultados que se desejam alcançar em conjunto (AGUIAR, 2014). Isso irá facilitar também as auditorias, pois consequentemente os dados gerados por uma gestão participativa é divulgado com maior credibilidade, pois desde sua elaboração, presume-se que tenha seguido todas as normas vigentes na área ambiental (BARBIERI, 2007; GONÇALVES et al., 2015).

O gestor pode propor alternativas como a decisão da criação de estacionamentos para bicicletas em todos os edifícios, motivando os seus membros a trocar seus carros por bicicletas, assim como outras iniciativas sustentáveis, sabendo que os resultados serão heterogêneos em decorrências das necessidades e atividades realizadas em cada setor da instituição. No entanto, sem perder de vista o objetivo geral que é a preservação ambiental e a realização de todas as atividades com o menor consumo possível (ALAÑACASTILLO et al., 2017; CREPALDI, 2013; SEIFFERT, 2010).

Deixando evidente em suas ações que o desenvolvimento sustentável é alcançar suas metas estabelecias sem comprometer a disponibilidade de insumos futuro. As IES tem um papel fundamental a ser considerado devido ser geradora de tecnologia, porém em muitos casos não se responsabiliza pelos resíduos que faturamento essa tecnologia irá se tornar, que foge do papel da instituição que assume apenas a responsabilidade social que é educar e deixar sua marca no futuro de profissionais certificados por ela (FERREIRA et al., 2017; FERIGATO, 2020; RAMÍREZ-OSPINA et al., 2017; SANTOS, 2017). Assim, ressaltar-se que a pesquisa acadêmica afeta os seres humanos e o meio ambiente, e as IES são responsáveis por seus objetivos e resultados.

\section{CONCLUSÕES}

É papel de todos preservar o meio ambiente, e os membros da instituição desempenham a responsabilidade maior perante a sociedade pela função que desempenham nesse contexto. É possível se alcançar estágios sustentáveis em uma instituição quando os membros identificam-se com os objetivos de proteção ambiental e desenvolvimento sustentável, e colaboraram com a implementação destes.

A proteção do meio ambiente significa impedir processos que possam ocasionar riscos para as pessoas e a perpetuação de recursos naturais, realizado através da diminuição do uso de recursos, da energia, da minimização de emissões de resíduos, e para isso as IES deverá construir um ambiente propicio para efetuar esses objetivos. E os departamentos institucionais devem estar vinculados para que o gestor alcance as metas definidas.

As IES dever-se comprometer a informar a comunidade sobre as medidas de proteção de forma transparente e fornecer a educação, treinamento e incentivo aos seus colaboradores em relação à preservação do meio ambiente e das possíveis soluções de problemas ambientais. E de forma participativa antes da aquisição de insumos verificar com a comunidade as reais necessidades dos mesmos, dessa forma os efeitos ambientais ocasionados por materiais e equipamentos devem ser levados em consideração pela instituição antes da compra.

Devendo prioritariamente as IES desempenhar atividades ambientais relevantes e garantir a 
liberdade na pesquisa e ensino, conscientizando a comunidade de sua responsabilidade social e das consequências ambientais que certos resíduos podem ocasionar. Por último, enfatizar-se a importância de se preservar os recursos naturais para as gerações presentes e futuras, e a promoção da busca de auxilio de colaboradores e discentes, com a finalidade de minimizar as consequências negativas na pesquisa e no ensino institucional, garantindo assim, a maior oferta de trabalhos acadêmicos na área ambiental e cursos que tratam das questões ambientais, em cooperação com outros centros de pesquisas.

\section{REFERÊNCIAS}

AGUIAR, C.. Ranking internacional destaca UFLA como a 26a universidade mais sustentável do mundo. Universidade Federal de Lavras, 2015.

AGUIAR, C.. Técnicos da Cemig visitam a UFLA para apresentação da Usina Solar Fotovoltaica. Universidade Federal de Lavras, 2014.

ALAÑA CASTILLO, T. P.; MORÁN MOLINA, G. G.; SANMARTÍN RAMÓN, G. S.. La auditoría ambiental en las MIPYMES como herramienta de control interno en la gestión empresarial. Revista Universidad y Sociedad, v.9, n.1, p.143-147, 2017.

ALLAM, H.; KUEHR, R.; HUISMAN, J.; GOSSART, C.. StepSolving the e-waste problem. United Nations University, Mauritius, 2011.

ALONSO-ALMEIDA, M. M.; MARIMON, F.; CASANI, F.; RODRIGUEZ-POMEDA, J.. Diffusion of sustainability reporting in universities: current situation and future perspectives. Journal of Cleaner Production, v.106, p.144-154, 2015. DOI: https://doi.org/10.1016/j.jclepro.2014.02.008

BARBIERI, J. C.. Gestão ambiental empresarial: conceitos, modelos e instrumentos. 2 ed. São Paulo: Saraiva, 2007.

BOCKEN, N.; SHORT, S., RANA; P.; EVANS, S.. A literature and practice review to develop sustainable business model archetypes. Journal of Cleaner Production, v.65, p.42-56, 2014. DOI: https://doi.org/10.1016/j.jclepro.2013.11.039

BOFF, L.. Sustentabilidade: tentativa de definição. Petrópolis: Vozes, 2012.

BRANDLI, L. L.; FRANDOLOSO, M. A. L.; FRAGA, K. T.; VIEIRA, L. C.; PEREIRA, L. A.. Avaliação da presença da sustentabilidade ambiental no ensino dos cursos de graduação da Universidade de Passo Fundo. Avaliação, v.17, n.2, p.433-454, 2012. http://dx.doi.org/10.1590/S141440772012000200008 .

BRASIL. Constituição da República Federativa do Brasil. Brasília: DOU, 1988.

BRIDGESTOCK, L.. Green Universities. Top Universities, 2012.

BRUSEKE, F. J.. Desestruturação e desenvolvimento. In: VIOLA, E.; FERREIRA, L. C.. Incertezas de sustentabilidade na globalização. Campinas: Unicamp, 1996.

CREPALDI, S. A.. Auditoria Contábil: teoria e prática. 9 ed. São Paulo: Atlas, 2013.
DUTTA, S. K.; LAWSON, R. A.; MARCINKO, D. J.. A management control system to support corporate sustainability strategies. Advances in Accounting, v.32, p.1017, 2016. DOI: https://doi.org/10.1016/j.adiac.2015.12.001

EL FARO, O.; CALIA, R. C.; PAVAN, V. H. G.. A logística reversa do lixo tecnológico: um estudo sobre a coleta do e-lixo em uma importante universidade brasileira. Revista de Gestão Social e Ambiental, v.6, n.3, p.142-153, 2012. DOI: http://dx.doi.org/10.5773/rgsa.v6i3.461

FERIGATO, E.; CONCEIÇÃO, M. M.; ROSINI, A.M.; CONCEIÇÃO, J. T. P.. Auditoria ambiental e sua importância como ferramenta de gestão ambiental. Research, Society and Development, v.9, n.8, p.1-34, 2020. DOI: https://doi.org/10.33448/rsd-v9i8.6569.

FERREIRA, E. C.; RODRIGUES, W. C.. A Relação entre o Impacto Ambiental em Mariana (MG) e o Resultado Econômico e Financeiro da Empresa Mineradora Samarco S/A. Instituto de Ensino Superior Blauro Cardoso de Mattos, 2017.

FREITAS, C. C. G.; MAÇANEIRO, M. B.; KUHL, M. R.; SEGATTO, A. P.; DOLIVEIRA, S. L. D.; LIMA, L. F.. Transferência tecnológica e inovação por meio da sustentabilidade. RAP Revista de Administração Pública, v.46, n.2, p.363-384, 2012.

GARCIA, S.; CINTRA, Y.; TORRES, R. C..; LIMA, F. G.. Corporate sustainability management: a proposed multi-criteria model to supportbalanced decision-making. Journal of cleaner production, v.136, p.181-196, 2016. DOI: https://doi.org/10.1016/j.jclepro.2016.01.110.

GIL, A. C.. Como elaborar projetos de pesquisa. 4 ed. São Paulo: Atlas, 2002.

GONÇALVES, S. R.; MENDES, L. R. S.. O controle na administração pública: o papel da auditoria interna em uma instituição federal de ensino superior de Minas Gerais. Cadernos Zygmunt Bauman, v.5, n.9, 2015.

INEP. Indicadores de Qualidade da Educação Superior. Brasília: INEP, 2018.

INSTITUTO HUMANISTA UNISINOS.. A crise do setor energético e a síndrome de país rico. Entrevista especial com Luiz Augusto Horta Nogueira, 2014.

JORGE, M. L.; MADUEÑO, J. H.; CEJAS, M. Y. C.; PEÑA, F. J. A.. An approach to the implementation of sustainability practices in Spanish universities. Journal of Cleaner Production, v.106, p.34-44, 2015. 
LAKATOS, E. M.; MARCONI, M. A.. Fundamentos de Metodologia Científica. 5 ed. São Paulo: Atlas, 2003.

MAYOR, F.. Preparar um futuro viável: ensino superior e desenvolvimento sustentável. In: CONFERÊNCIA MUNDIAL SOBRE O ENSINO SUPERIOR. Anais. Paris, 1998.

BRASIL. Portaria № 23. Estabelece boas práticas de gestão e uso de energia elétrica e de águas nos órgãos e entidades da Administração Pública Federal direta, autárquica e fundacional e dispõe sobre o monitoramento de consumo desses bens e serviços. Brasília: DOU, 2015.

MIRANDA, E. P. P.. Proposta para utilização de sistemas automatizados em universidades para economia de energia elétrica. Monografia (Graduação em Engenharia Eletrônica) - Universidade Tecnológica Federal do Paraná, Ponta Grossa, 2017.

OSIBANJO, O., NNOROM, I. C.. The challenge of electronic waste (e-waste) management in developing countries. Waste Management \& Research, v.25, p.489-501, 2007. DOI: https://doi.org/10.1177/0734242X07082028

PRYSHLAKIVSKY, J., SEARCY, C.. Fifteen years of ISO 14040: a review. Journal of Cleaner Production, v.57, p.115-123, 2013. DOI: http://doi.org/10.1016/i.jclepro.2013.05.038

RAMÍREZ OSPINA, D. E.; ANDRADE ARANGO, Y.. Aporte de la contabilidad y la auditoría desde una perspectiva ambiental. Textual: análisis del medio rural latinoamericano, v.69, n.2741, 2017.

RINGSTRÖM, E.; WIDHEDEN, J.. Some definitions of Ecoefficiency. 2011.

ROGERS, D. S.; TIBBEN-LEMBKE, R. S.. An examination of reverse logistics practices. Journal of Business Logistics, v.22, n.2, p.129-148, 2001. DOI: https://doi.org/10.1002/j.2158-1592.2001.tb00007.x
SALGADO, M. M. A.. Desenvolvimento de programa de gestão ambiental para Instituições de Ensino Superior: estudo de caso - Instituto Esperança de Ensino Superior. Dissertação (Mestrado em Sistemas de Gestão) Universidade Federal Fluminense: Niterói, 2006.

SANTOS, N. K. F. S.. Auditoria ambiental como ferramenta de confirmação da sustentabilidade empresarial: um estudo sobre a sua utilização nas empresas listadas no índice de sustentabilidade empresarial - ISE/BOVESPA de 2010 a 2015. Monografia (Bacharelado em Ciências Contábeis) Universidade Federal de Campina Grande: Sousa, 2017.

SEIFFERT, M. E. B.. Gestão ambiental: instrumentos, esferas de ação e educação ambiental. São Paulo: Atlas, 2010.

SHARP, L.. Green campuses: the road from little victories to systemic transformation. International Journal of Sustainability in Higher Education, v.3, n.2, p.128-145, 2002. DOI: https://doi.org/10.1108/14676370210422357

UNHABITAT. United Nations Human Settlements Programme. Urbanization and development: emerging futures. 2016.

WIDMER, R.; OSWALD-KRAPF, H.; SINHA-KHETRIWAL, D., SCHNELLMANN, M.; BONI, H.. Global perspectives on ewaste. Environmental Impact Assessment Review, v.25, p.436-458, 2005. https://doi.org/10.1016/i.eiar.2005.04.001

WOLSKI, G. D. M.. E-waste and the sustainable organisation: Griffith University's approach to e-waste". International Journal of Sustainability in Higher Education, v.10, n.1, p.21-32, 2009. DOI: http://dx.doi.org/10.1108/14676370910925226

ZANELLA, L. C. H.. Metodologia de Estudo e de Pesquisa em Administração. 2 ed. Florianópolis: UFSC, 2012.

A CBPC - Companhia Brasileira de Produção Científica (CNPJ: 11.221.422/0001-03) detém os direitos materiais desta publicação. Os direitos referem-se à publicação do trabalho em qualquer parte do mundo, incluindo os direitos às renovações, expansões e disseminações da contribuição, bem como outros direitos subsidiários. Todos os trabalhos publicados eletronicamente poderão posteriormente ser publicados em coletâneas impressas sob coordenação da Sustenere Publishing, da Companhia Brasileira de Produção Científica e seus parceiros autorizados. Os (as) autores (as) preservam os direitos autorais, mas não têm permissão para a publicação da contribuição em outro meio, impresso ou digital, em português ou em tradução. 